\title{
The entropic pressure of lattice knots
}

\author{
E.J. Janse van Rensburg \\ Department of Mathematics and Statistics, York University \\ Toronto, Ontario, M3J 1P3, Canada
}

October 12, 2018

\begin{abstract}
The entropic pressure in the vicinity of a cubic lattice knot is examined as a model of the entropic pressure near a knotted ring polymer in a good solvent. A model for the scaling of the pressure is developed and this is tested numerically by sampling lattice knots using a Monte Carlo algorithm. Good agreement is found between scaling predictions and numerical experiments.
\end{abstract}

\section{Introduction}

A polymer placed close to a geometric obstacle (such as a hard wall) loses conformational entropy and this induces a net force on the obstacle [27]. This induced force is repulsive and exerts an average net pressure on the wall. Entropically induced forces have been observed experimentally $[4,6]$, and have been simulated using self-avoiding walk models of a grafted polymer [24].

A slightly different situation is encountered when a (small) test particle is placed close to a polymer - see figure 1 for a schematic illustration. The volume occupied by the particle excludes volume, and this causes a loss of conformational entropy in the polymer. The consequence is that the particle experiences a net repulsive force - if it is free to move, then it will be expelled from the vicinity of the polymer. An effect like this underlies the polymeric stabilisation of a colloid [32].

The forces induced on a test particle near a polymer may be described as a gradient of a pressure field in the vicinity of the polymer. This was examined in several studies (see for example references $[3,14,28]$, and reference [5] for a directed lattice path model).

In reference [13] a two dimensional self-avoiding walk model of a ring polymer (a square lattice polygon [16]) was used to simulate the entropically induced pressure near a two dimensional lattice polygon model of a ring polymer. A scaling analysis for the net pressure was done and used to determine the pressure as a function of distance from the polygon.

In particular, let $R=\langle r\rangle_{n}$ be a length scale in the model (such as the root mean square radius of gyration or mean span of a polygon of length $n$ ). Then $R \sim n^{\nu}$ 


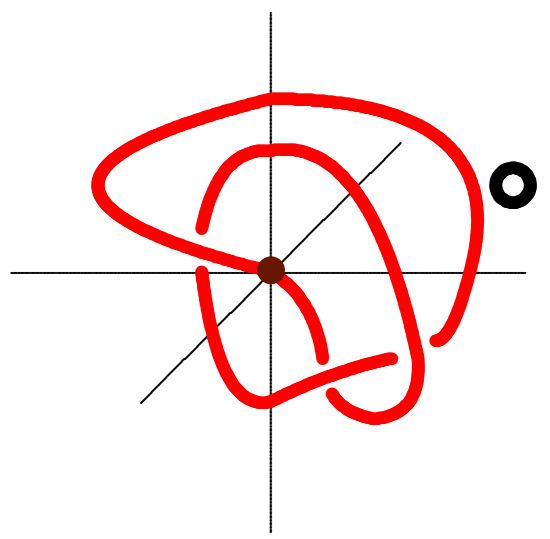

Figure 1: Schematic illustration of a test particle near a ring polymer in three dimensions. The particle is an obstacle which limits the conformational degrees of freedom of the polymer. This reduction in polymer entropy induces a repulsive net force on the particle. This force may be described as the gradient of an average pressure field in the vicinity of the polymer.

where $\nu$ is the metric exponent of the self-avoiding walk. In two dimensions the pressure $\mathbf{P}_{n}(a)$ at a distance $a R$ from the polygon, for $a>0$, was shown to scale as

$$
\mathbf{P}_{n}(a) \simeq C g(a) a^{-2 d} n^{2 \gamma_{1}-1-4 \nu-\alpha_{s}+2}
$$

where $\gamma_{1}$ is the entropic exponent of self-avoiding walks in a half-space [19], $\alpha_{s}$ is the entropic exponent of lattice polygons [11], and $g(a)$ is a scaling function of the general form

$$
g(a)=\int_{0}^{1} x^{\gamma_{1}-1}(1-x)^{\gamma_{1}-1} f\left(a / x^{\nu}\right) f\left(a /(1-x)^{\nu}\right) d x .
$$

The function $f(x)$ is a quickly decaying function (the choice $f(x)=e^{-x}$ proved consistent with numerical data).

Using the exact values in $d=2$ dimensions in equation (1) gives

$$
n^{19 / 32} \mathbf{P}_{n}(a) \simeq C g(a) a^{-4} .
$$

This result gave excellent agreement with numerical data [13].

In this paper the pressure near a lattice model of a ring polymer in three dimensions is examined. The model is illustrated schematically in figure $1-$ a particle is placed near a lattice polygon to measure the pressure which may scale in a way similar to the two dimensional result in equation (1). Substitution of estimated values for exponents in $d=3$ dimensions gives $n^{0.193} P_{n}(a) \simeq C g(a) a^{-6}$. Preliminary simulations of three dimensional lattice polygons showed that this prediction is inconsistent with numerical data. 
In other words, the scaling relation for square lattice polygons in equation (1) must be modified for cubic lattice polygons. This is done in section 2 , and the resulting relation is tested numerically in section 3 . The rescaled pressure $\mathbf{P}_{n}(a)$ for lattice polygons in $d \geq 3$ dimensions is shown to be

$$
\mathbf{P}_{n}(a) \simeq C n^{\gamma-2 d \nu-\alpha_{s}+2} g_{d}(a) a^{-2 d} \text { in } d \geq 3 \text { dimensions, }
$$

where $g_{d}(a)$ is given by

$$
g_{d}(a)=\int_{0}^{1} G\left(a / x^{\nu}\right) G\left(a /(1-x)^{\nu}\right) d x .
$$

As in the definition of $g(a)$ above, the function $G(x)$ is a quickly decaying function, and choosing an exponential works well. More properly, results in references $[8,9]$ suggest that for large values of $x$ it should be expected that $G(x) \sim x^{d} e^{-x^{\delta}}$ where $\delta=1 /(1-\nu)$; see section 2.2 .

The hyperscaling relation $2-\alpha_{s}=d \nu$ for polygons in low dimensions can be used to obtain

$$
n^{d \nu-\gamma} \mathbf{P}_{n}(a) \simeq C g_{d}(a) a^{-6} \text { if } d=3 .
$$

For $d>4$ the hyperscaling relation breaks down, and the relation in equation (4) should be used instead.

Substituting three dimensional estimated values for $\gamma$ and $\nu[7,34]$ in the above gives $3 \nu-\gamma \approx 0.606$ so that

$$
n^{0.606} \mathbf{P}_{n}(a) \approx C g_{d}(a) a^{-6} \text { in } d=3 \text { dimensions. }
$$

This result is quite different from the prediction in equation (1). In section 2 it will be seen why a different result is obtained in 3 dimensions, compared to the result derived for 2 dimensions in reference [13].

In section 2 the derivation of the scaling relation for $\mathbf{P}_{n}(a)$ in equation (6) is presented for lattice polygons in the hypercubic lattice (with $d \geq 3$ ). This derivation is similar to that of equation (1), but differs in a few important ways due to the different dimensionality. Equation (1) was derived in the square lattice where steric interactions of the polygon with itself is an important factor. A different set of constraints (or rather the lack thereof) in the cubic lattice (or higher dimenionsonal lattices) gives a different result if $d \geq 3$.

Since lattice polygons in the cubic lattice are embeddings of the circle in three space, they have well defined knot types. Cubic lattice polygons are lattice knots, and they are models of knotted ring polymer entropy. In section 3 it is argued that the scaling analysis of section 2 is valid for rooted lattice knots as well. This is tested in section 3 by Monte Carlo simulations of lattice knots. The sampling of lattice knots was done by using an implementation of the GAS-algorithm for lattice polygons in the cubic lattice $[20,21]$ (implemented with BFACF elementary moves $[1,2])$. Data were collected in several different simulations for lattice knots of types $K \in\left\{0_{1}, 3_{1}, 4_{1}, 5_{1}, 5_{2}\right\}$ (where $0_{1}$ is the unknot, $3_{1}$ is the trefoil, and 


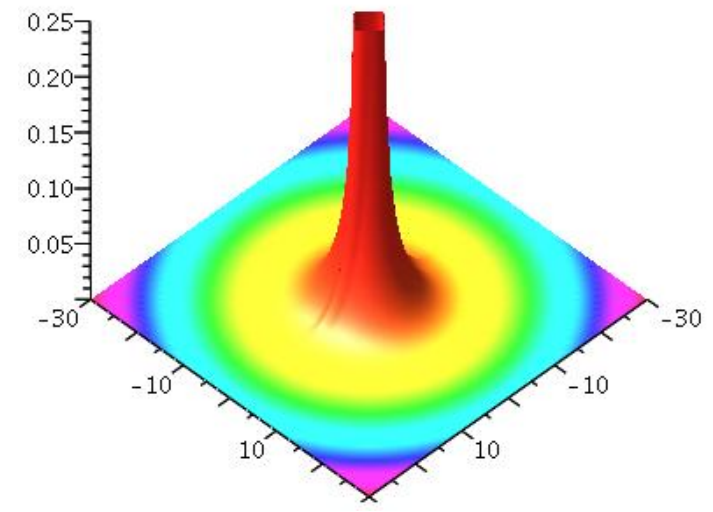

Figure 2: The pressure field near a lattice knot of type unknot. The polygons are rooted at the origin and are of length 100 . The units on the horizontal axes are in lattice steps. The pressure peaks sharply towards the origin, and decays to zero with increasing distance. It is isotropic around the origin up to small corrections close to the origin.

$4_{1}$ is the figure eight knot). For each knot type the calculated pressure should have scaling given by equation (7). The numerical data confirm this expectation. For example, the data show that the pressure is similar for different knot types, deviating only at long distances from one another. In each case the estimated pressure exhibits scaling consistent with equation (7).

The numerical data also show that the pressure field near a rooted lattice knot is isotropic (independent of direction from the origin). This result is consistent with the results in reference [13]. For example, in figure 2 the calculated pressure field in the $x y$-plane near a lattice unknot is plotted for polygons of length $n=100$. The pressure increases sharply as the origin is approached (since the polygon is rooted at the origin, the pressure at this point is undefined). The calculated and interpolated pressure field, even at small distances from the origin, is isotropic within numerical error (and with some residual effects due to the underlying lattice). This result is also supported by the rescaled data, as will be shown.

The paper is concluded with a few final observations in section 4.

\section{Scaling of the entropic pressure}

Denote the standard basis of $\mathbb{R}^{3}$ by $\left(\vec{e}_{1}, \vec{e}_{2}, \vec{e}_{3}\right)$. Denote the cubic lattice by $\mathbb{L}^{3}$ and let its origin be $\overrightarrow{0}$. Then $\mathbb{L}^{3}$ is a graph with vertices $\vec{v} \in \mathbb{Z}^{3}$ and edges $\vec{v} \sim \vec{W}$ joining vertices $\vec{v}$ and $\vec{w}$ if $\|\vec{v}-\vec{w}\|_{2}=1$. 
A self-avoiding walk of length $n$ from the origin in $\mathbb{L}^{3}$ is a sequence of edges $\left\langle\vec{v}_{i} \sim \vec{v}_{i+1}\right|$ for $\left.i=0,1, \ldots, n-1\right\rangle$, where the $\vec{v}_{i}$ are distinct and $\vec{v}_{0}=\overrightarrow{0}$. Walks are oriented from $\overrightarrow{0}$. The number of self-avoiding walks of length $n$ edges from $\overrightarrow{0}$ is a function denoted by $c_{n}$. It is known that the limit

$$
\lim _{n \rightarrow \infty} \frac{1}{n} \log c_{n}=\log \mu
$$

exists (see reference [17]), and $\kappa=\log \mu$ is the connective constant of the selfavoiding walk. This shows that $c_{n}=\mu^{n+o(n)}-$ and $\mu$ is called the growth constant.

A self-avoiding walk $\omega=\left\langle\vec{v}_{0} \sim \vec{v}_{1}, \vec{v}_{1} \sim \vec{v}_{2}, \ldots, \vec{v}_{n-1} \sim \vec{v}_{n}\right\rangle$ is a lattice polygon if $\vec{v}_{0}=\vec{v}_{n}$. Notice that lattice polygons are not oriented (by convention). Two lattice polygons are equivalent if the first is a translation of the second in $\mathbb{L}^{d}$. Let $p_{n}$ be the number of distinct lattice polygons of length $n$. For example, if $d=3$, then it may be checked that $p_{4}=3, p_{6}=22$ and $p_{8}=207$. Since $\mathbb{L}^{d}$ is bipartite it follows that $p_{2 n+1}=0$. It is known that the limit

$$
\lim _{n \rightarrow \infty} \frac{1}{n} \log p_{n}=\log \mu
$$

exists in $\mathbb{L}^{d}$ (if the limit is taken through even values of $n$ ), and $\mu$ is the growth constant of self-avoiding walks defined in equation $(8)[16,18]$. Lattice polygons in $\mathbb{L}^{d}$ are models of ring polymer entropy [16].

A lattice polygon $\omega$ is rooted at $\overrightarrow{0}$ in $\mathbb{L}^{d}$ if it contains the origin. The number of rooted polygons in $\mathbb{L}^{d}$ is $\bar{p}_{n}=n p_{n}$, since there are $n$ vertices in $\omega$ which can be placed at the origin.

The number of self-avoiding walks has asymptotic behaviour

$$
c_{n} \simeq A n^{\gamma-1} \mu^{n}
$$

where $\gamma$ is the entropic exponent of the self-avoiding walk. The number of lattice polygons of length $n$ has asymptotic behaviour

$$
p_{n} \simeq B n^{\alpha_{s}-3} \mu^{n}
$$

where $\alpha_{s}$ is the entropic exponent of lattice polygons. This shows that $\bar{p}_{n} \simeq$ $B n^{\alpha_{s}-2} \mu^{n}$.

The exponents $\gamma$ and $\alpha_{s}$ have been estimated in the literature. For example, the exponent $\gamma$ can be accurately obtained from field theoretic calculations in three dimensions. Such calculations gave the very accurate estimate

$$
\gamma=1.15698 \pm 0.00034 \text {, in reference [25]. }
$$

The entropic exponent of lattice polygons in three dimensions was estimated to have value

$$
\alpha_{s}=0.237 \pm 0.002 \text {, in reference [15]. }
$$




\subsection{The conformational entropy of lattice polygons}

Let $\bar{p}_{n}(\vec{r})$ be the number of polygons rooted at $\overrightarrow{0}$ passing through the point $\vec{r}=$ $(x, y, z)$ in $\mathbb{L}^{d}$. Then $\widetilde{p}_{n}(\vec{r})=\bar{p}_{n}-\bar{p}_{n}(\vec{r})$ is the number of rooted lattice polygons avoiding the lattice site $\vec{r}$.

The conformational entropy of a polygon rooted at the origin is given by $S_{n}=$ $k_{B} \log \bar{p}_{n}$, where $k_{B}$ is Boltzmann's constant. The entropy of lattice polygons avoiding $\vec{r}$ is $\widetilde{S}_{n}(\vec{r})=k_{B} \log \widetilde{p}_{n}(\vec{r})$. Thus, the change in entropy if the site $\vec{r}$ is excluded (or if a hard test particle is placed at $\vec{r}$ ) is given by

$$
\Delta S_{n}(\vec{r})=k_{B} \log \widetilde{p}_{n}(\vec{r})-k_{B} \log \bar{p}_{n}
$$

The change in extensive free energy is given by $\Delta F_{n}(T)=-T \Delta S_{n}(\vec{r})$. Thus, the entropic pressure at the site $\vec{r}$ can be computed by considering the change in $F_{n}(T)$ due to a change in volume (by excluding the lattice site at $\vec{r}$ ). This is given by

$$
P_{n}(\vec{r})=\frac{\Delta F_{n}(T)}{\Delta V(\vec{r})}=-T \frac{\Delta S_{n}(\vec{r})}{\Delta V(\vec{r})} .
$$

Choose units so that $T=k_{B}=1$ and let $\Delta V(\vec{r})$ be the (unit square or cubical) volume element with $\vec{r}$ at its centre (so that $|\Delta V(\vec{r})|=1$ ). By equation (14) it follows that the pressure at the point $\vec{r}$ is given by

$$
P_{n}(\vec{r})=\log \bar{p}_{n}-\log \widetilde{p}_{n}(\vec{r})=-\log \left(1-\frac{\bar{p}_{n}(\vec{r})}{\bar{p}_{n}}\right) .
$$

$P_{n}(\vec{r})$ is non-negative.

Rescale $P_{n}(\vec{r})$ as follows. Let $R_{n}^{2}=\left\langle r^{2}\right\rangle_{n}$ be the mean square radius of gyration of rooted lattice polygons of length $n$. Define the rescaled vectors $\vec{c}_{r}=\vec{r} / R_{n}$. Then $a \vec{c}_{r}$ is a vector parallel to $\vec{r}$ for $a>0$. If $a=1$ then $\|\vec{r}\|_{2}=R_{n}$, so that the units of $a$ is $R_{n} \simeq C n^{\nu}$.

Put $\mathbf{P}_{n}(a)=P_{n}\left(a \vec{c}_{r} R_{n}\right)$. Then $\mathbf{P}(1)$ is the pressure at a point a distance $R_{n}$ from $\overrightarrow{0}$ in the direction $\vec{c}_{r}$. Assuming that $P_{n}(\vec{r})$ is isotropic shows that $\mathbf{P}_{n}(a)$ is the pressure at a distance $a R_{n}$ from $\overrightarrow{0}$. There are corrections to this assumption for short polygons, but the data will show that this is valid for longer polygons, subject to small corrections due to the geometry of the underlying lattice. This defines the rescaled pressure $\mathbf{P}_{n}(a)$ in section 1 , and in equations (1) and (6).

\subsection{Metric properties of walks and polygons}

The number of self-avoiding walks of length $n$ steps from $\overrightarrow{0}$ in $\mathbb{L}^{3}$ to a vertex $\vec{r}$ is $c_{n}(\vec{r})$. Define $c_{n}(r)=\sum_{|\vec{r}|=r} c_{n}(\vec{r})$ to be the number of walks which ends at a distance $r$ from $\overrightarrow{0}$ after $n$ steps. Since such walks end on a spherical shell of radius $r$, it is expected that

$$
c_{n}(r) \simeq A_{0} r^{2} c_{n}(\vec{r})
$$

in the cubic lattice $\mathbb{L}^{3}$. 
The average distance of the endpoint of a self-avoiding walk of length $n$ from the origin is denoted by $\langle r\rangle_{n}$, and this introduces a metric in the model. The expected distance of the endpoint of the walk from $\overrightarrow{0}$ can be computed from $c_{n}(r)$ by

$$
R_{c}=\langle r\rangle_{n}=\frac{1}{c_{n}} \sum_{r \geq 0} r c_{n}(r) \simeq C n^{\nu}
$$

where $\nu$ is the metric exponent of the self-avoiding walk. The metric exponent is known to high accuracy in three dimensions:

$$
\nu=0.587597 \pm 0.000007 \text { in reference [7] }
$$

The ratio $c_{n}(\vec{r}) / c_{n}$ is the (spherically symmetric) end-to-end distribution function $P_{n}(r)$ of self-avoiding walks [12], and it is the probability that a walk of length $n$ has endpoint a distance $r=|\vec{r}|$ from the origin in the direction of $\vec{r} . P_{n}(r)$ has behaviour given by

$$
P_{n}(r) \sim R_{c}^{-d} F_{0}\left(\frac{r}{R_{c}}\right)
$$

That is, it is the inverse volume occupied by the end point of the walk (namely $R_{c}^{-d}$ ) multiplied by a modifying scaling function $F_{0}$ which is a function of the distance from the origin scaled by (the length scale) $R_{c}$. $F_{0}$ has the following conjectured properties [8]:

$$
F_{0}(x) \sim x^{g}, \text { for small } x \text { and } F_{0}(x) \sim e^{-x^{\delta}} \text { for large } x .
$$

where $g=\frac{\gamma-1}{\nu}[8,9]$ and $\delta=\frac{1}{1-\nu}[12]$.

Thus, in terms of $P_{n}(r)$,

$$
c_{n}(\vec{r}) \simeq P_{n}(r) c_{n} \sim \frac{1}{R_{c}^{d}} F_{0}\left(\frac{r}{R_{c}}\right) c_{n}=r^{-d}\left(\frac{r}{R_{c}}\right)^{d} F_{0}\left(\frac{r}{R_{c}}\right) c_{n} .
$$

Put $G(x)=x^{d} F_{0}(x)$, then the above becomes

$$
c_{n}(\vec{r}) \approx C_{0} r^{-d} G\left(\frac{r}{R_{c}}\right) c_{n} .
$$

Notice that $R_{c} \sim R_{n} \simeq C n^{\nu}$, where $R_{n}^{2}$ is the mean square radius of gyration of lattice polygons.

\subsection{The number of polygons passing through a lattice point $\vec{r}$}

The pressure at a point $\vec{r}$ near a lattice polygon rooted at $\overrightarrow{0}$ in $\mathbb{L}^{d}$ with $d \geq 3$ is computed by estimating the number of polygons passing through $\vec{r}$. The basic approach is to consider each such polygon as being composed of two walks from $\overrightarrow{0}$ to $\vec{r}$. The number of such pairs of walks will be estimated by using equation (23).

Denote the number of lattice polygons rooted at $\overrightarrow{0}$ in $\mathbb{L}^{d}$, of length $n$, containing the lattice point $\vec{r}$ by $\bar{p}_{n}(\vec{r})$. Then $\bar{p}_{n}(\vec{r})$ can be approximated by a pair of walks from $\overrightarrow{0}$ and ending in $\vec{r}$. If these walks avoid one another, then their union is a 
rooted lattice polygon containing $\vec{r}$. If they do not avoid one another, then an upper bound on $\bar{p}_{n}(\vec{r})$ is obtained.

This shows that

$$
\bar{p}_{n}(\vec{r}) \simeq \sum_{k=0}^{n} P_{k, n-k} c_{k}(\vec{r}) c_{n-k}(\vec{r})
$$

where $P_{k, n-k}$ is the probability that a walk of length $k$ and a walk of length $n-k$ from $\overrightarrow{0}$ to $\vec{r}$ avoid one another.

The summand in equation (24) can be estimated by using equation (23). This replaces the functions $c_{k}(\vec{r})$ and $c_{n-k}(\vec{r})$ in the summand of equation (24) by factors containing $c_{k}$ and $c_{n-k}$.

The probability $P_{k, n-k}$ is estimated as follows. Let $Q_{k, n-k}(\vec{r}, \vec{s})$ be the probability that a walk of length $k$ from $\overrightarrow{0}$ to $\vec{r}$ intersects a walk of length $n-k$ from $\overrightarrow{0}$ to $\vec{s}$. Since such walks are likely to intersect near $\overrightarrow{0}$ (where they start, irrespective of the choices for $\vec{r}$ and $\vec{s}$ ), this probability is to leading order given by the probability that two walks, of lengths $k$ and $n-k$, intersect each other. That is, to leading order $Q_{k, n-k}(\vec{r}, \vec{s})$ should be only weakly dependent on $\vec{r}$ and $\vec{s}$. In other words, $P_{k, n-k}$ may be approximated by the probability that two walks from $\overrightarrow{0}$, of lengths $k$ and $n-k$ avoid one another. Denote this probability by $P_{k, n-k}^{r}$. This gives the following estimate for $\bar{p}_{n}(\vec{r})$ :

$$
\bar{p}_{n}(\vec{r}) \simeq C_{0}^{2} r^{-2 d} \sum_{k=0}^{n} P_{k, n-k}^{r} G\left(r / C k^{\nu}\right) G\left(r / C(n-k)^{\nu}\right) c_{k} c_{n-k}
$$

$P_{k, n-k}^{r}$ is given by $P_{k, n-k}^{r}=c_{n} /\left(c_{k} c_{n-k}\right)$. Thus, substituting $P_{k, n-k}^{r}$,

$$
\bar{p}_{n}(\vec{r}) \simeq A_{1} C_{n} r^{-2 d} \sum_{k=0}^{n} G\left(r / C k^{\nu}\right) G\left(r / C(n-k)^{\nu}\right)
$$

for some constant $A_{1}=C_{0}^{2}$.

Introduce the scaling factor a by putting $r=a R_{n}$ where $R_{n} \simeq C n^{\nu}$ is the root of the mean square radius of gyration of the polygon. Then $\vec{r}=\left(a R_{n}\right) \frac{\vec{r}}{|\vec{r}|}$ and $\bar{p}_{n}(\vec{r})$ becomes a function of $a$. Put

$$
\widehat{p}_{n}(a)=\bar{p}_{n}\left(\left(a R_{n}\right) \frac{\vec{r}}{|\vec{r}|}\right) .
$$

Then $\widehat{p}_{n}(a)$ is the number of polygons passing through a point a distance a $R_{n}$ from the origin, where $R_{n} \simeq C n^{\nu}$ defines the length scale in the problem. The result is that

$$
G\left(r / C k^{\nu}\right) \approx G\left(a\left(\frac{k}{n}\right)^{-\nu}\right) \text { and } G\left(r / C(n-k)^{\nu}\right) \approx G\left(a\left(1-\frac{k}{n}\right)^{-\nu}\right)
$$

in equation (26). 
Substitute $c_{n}$ by its scaling relation in equation (10), and put $A_{2}=A A_{1} C^{-2 d}$ to see that

$$
\widehat{p}_{n}(a) \simeq A_{2} n^{\gamma-1}\left(a n^{\nu}\right)^{-2 d} \mu^{n} \sum_{k} G\left(a\left(\frac{k}{n}\right)^{-\nu}\right) G\left(a\left(1-\frac{k}{n}\right)^{-\nu}\right) .
$$

Approximate the summation by an integral over $k$, and change variable $x=\frac{k}{n}$. This gives

$$
\widehat{p}_{n}(a) \simeq A_{2} n^{\gamma-2 d \nu} a^{-2 d} \mu^{n} \int_{0}^{1} G\left(a / x^{\nu}\right) G\left(a /(1-x)^{\nu}\right) d x .
$$

Define the function

$$
g_{d}(a)=\int_{0}^{1} G\left(a / x^{\nu}\right) G\left(a /(1-x)^{\nu}\right) d x .
$$

where $G(x)$ is given by $G(x)=x^{d} F_{0}(x)$ and $F_{0}(x)$ is defined in equation (21). Then

$$
\widehat{p}_{n}(a) \simeq A_{2} \mu^{n} n^{\gamma-2 d \nu} g_{d}(a) / a^{2 d}
$$

for some constant $A$. The dependence of $\widehat{p}_{n}(a)$ on $a$ is given by $g_{d}(a) / a^{2 d}$. This decays quickly to zero with increasing $a$. In figure 3 a plot of $g_{d}(a) / a^{2 d}$ against $a$ is shown on a logarithmic axes in $d=3$ dimensions. For small values of $a$ the graph decreases slowly on the log scale, but it turns over at about $a=1$ and then quickly decays to zero. This happens because of the super-exponential decay of the function $G(x)$ (see equation (21)).

\subsection{The rescaled pressure $\mathbf{P}_{n}(a)$}

By equation (16) the pressure due to rooted polygons at a point a distance $a R_{n}$ from the origin is approximately given by

$$
\mathbf{P}_{n}(a) \simeq-\log \left(1-\frac{\widehat{p}_{n}(a)}{\bar{p}_{n}}\right)
$$

where $\widehat{p}_{n}(a)$ is the number of polygons passing through a point a distance $a R_{n}$ from the origin and $\bar{p}_{n}=n p_{n}$ is the number of rooted polygons of length $n$.

The scaling of the pressure is obtained by using equations (30) and (11). In particular, for a not too small and for $n$ large this becomes

$$
\mathbf{P}_{n}(a) \simeq-\log \left(1-\frac{A_{2} g_{d}(a)}{B a^{2 d}} n^{\gamma-2 d \nu-\alpha_{s}+2}\right)
$$

since $\bar{p}_{n}=n p_{n}$. Since $\gamma-2 d \nu-\alpha_{s}+2<0$, and since $a^{-2 d} g_{d}(a)$ quickly approaches zero, the logarithm may be expanded. To leading order

$$
\mathbf{P}_{n}(a) \simeq \frac{A_{2} g_{d}(a)}{B a^{2 d}} n^{\gamma-2 d \nu-\alpha_{s}+2} .
$$




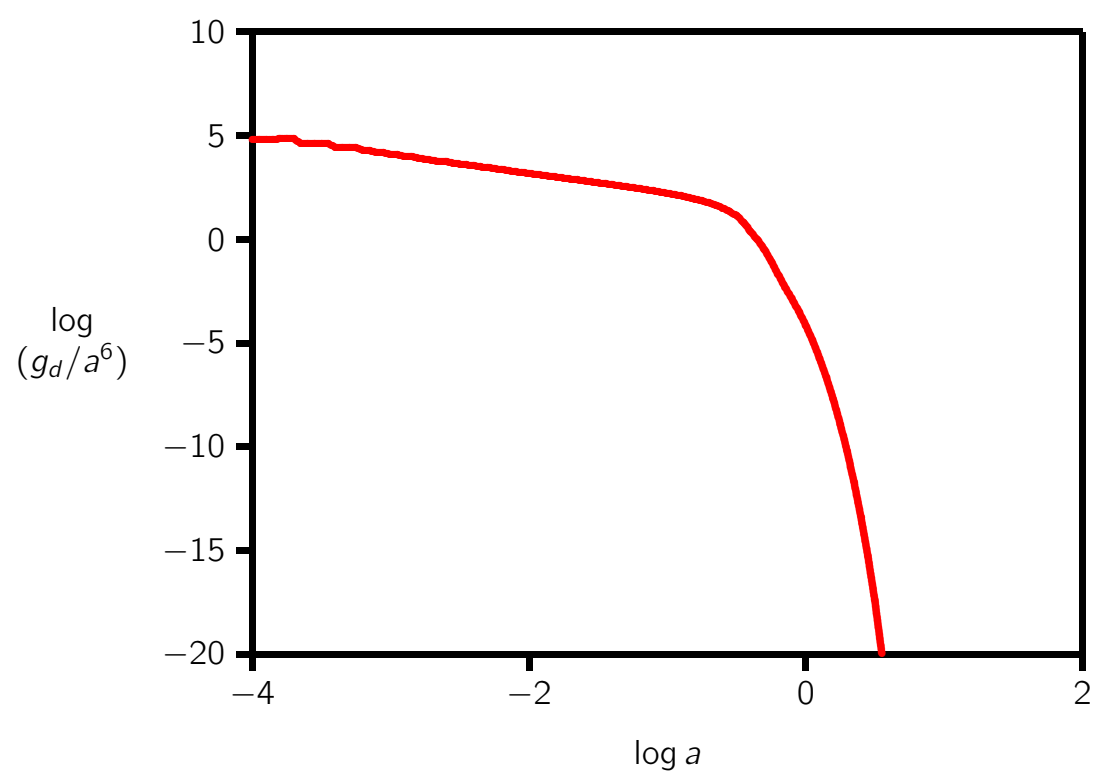

Figure 3: A plot of $\log \left(g_{d}(a) a^{-6}\right)$ against $\log a$.

The hyperscaling relation $2-\alpha_{s}=d \nu$ for polygons in three and four dimensions can be used to obtain

$$
\mathbf{P}_{n}(a) \simeq \frac{A_{2} g_{d}(a)}{B a^{2 d}} n^{\gamma-d \nu} .
$$

Using the approximate values $\gamma \approx 1.15$ and the Flory value $\nu=0.6$ gives $\mathbf{P}_{n}(a) \sim$ $n^{-0.65}$, while the more accurate estimates in equation (12) and (19) gives $\mathbf{P}_{n}(a) \sim$ $n^{-0.606}$. This gives the following relation in $d=3$ dimensions:

$$
n^{\rho} \mathbf{P}_{n}(a) \simeq C g_{d}(a) a^{-6},
$$

for some constant $C$, where the exponent $\rho$ should be approximately equal to 0.606 (and it is equal to $\gamma-d \nu$ in $d \geq 3$ dimensions - in contrast with its value in 2 dimensions given in equation (3)).

This prediction may be tested numerically by rescaling the lattice by $n^{-\nu}$ and the pressure by $n^{\rho}$ and then plotting $\left[n^{\rho} \mathbf{P}_{n}(a)\right]$ against $\left[|\vec{r}| / n^{\nu}\right]$. This should collapse data for a range of choices of $\vec{r}$ and $n$ to a single curve which is only a function of $a$ (and has the general shape shown in figure 3 ).

\section{The pressure near lattice knots}

Lattice polygons in $\mathbb{L}^{3}$ are piecewise linear embeddings of the circle in three space, and have well-defined knot type. For example, all polygons of length 4 are unknotted, and have knot type the unknot (denoted by $0_{1}$ ). It is known that all lattice polygons in $\mathbb{L}^{3}$ of length $n<24$ are unknotted [10]. 
Table 1: Numerical estimates of $\bar{p}_{n}$ and $\widetilde{p}_{n}(1,0,0)$ for $3_{1}$

\begin{tabular}{||r|ll|ll||}
\hline$n$ & $\bar{p}_{n}$ & $\sigma_{n}$ & $\widetilde{p}_{n}(1,0,0)$ & $\sigma_{n}$ \\
\hline \hline 24 & 33936 & 0 & 16797 & 21 \\
26 & $3.1067 \times 10^{6}$ & $0.0094 \times 10^{6}$ & $1.5230 \times 10^{6}$ & $0.0182 \times 10^{6}$ \\
28 & $1.7139 \times 10^{8}$ & $0.0091 \times 10^{8}$ & $8.4007 \times 10^{7}$ & $0.0102 \times 10^{7}$ \\
30 & $7.3863 \times 10^{9}$ & $0.0052 \times 10^{9}$ & $3.6367 \times 10^{9}$ & $0.0048 \times 10^{9}$ \\
32 & $2.7385 \times 10^{11}$ & $0.0024 \times 10^{11}$ & $1.3577 \times 10^{11}$ & $0.0018 \times 10^{11}$ \\
34 & $9.1671 \times 10^{12}$ & $0.0087 \times 10^{12}$ & $4.5805 \times 10^{12}$ & $0.0062 \times 10^{12}$ \\
\hline
\end{tabular}

Polygons in $\mathbb{L}^{3}$ of length 24 or longer may have non-trivial knot type. For example, polygons of knot type the trefoil (denoted by $3_{1}$ ) can be realised at length $n=24$ in $\mathbb{L}^{3}$ [10], and moreover, there are precisely 3328 distinct embeddings of length 24 which have knot type $3_{1}$ [33]. The minimal length of polygons of knot type $3_{1}$ in $\mathbb{L}^{3}$ is $n_{\min }\left(3_{1}\right)=24$. (Similarly, $n_{\min }\left(0_{1}\right)=4$ for the unknot). The knot type of the schematic ring polymer in figure 1 is a trefoil. If a polygon does not have knot type $3_{1}$ and its length is less than 30 , then its knot type is $0_{1}$ [33].

Lattice knots of type $4_{1}$ (the figure eight knot) can be realised with minimal length $n_{\min }\left(4_{1}\right)=30$, of type $5_{1}$ at $n_{\min }\left(5_{1}\right)=34$ and $5_{2}$ at $n_{\min }\left(5_{2}\right)=36-$ see for example references $[22,23,33]$ for more results.

The rescaled pressure $\mathbf{P}_{n}(a)$ for lattice polygons in equation (35) also applies to knotted polygons. To see this, notice that $\bar{p}_{n}(K)$, the number of lattice polygons of knot type $K$, rooted at $\overrightarrow{0}$, has been shown to have asymptotic growth given by

$$
\bar{p}_{n}(K) \approx B_{k} n^{\alpha_{s}-2+N_{k}} \mu^{n}
$$

where $N_{K}$ is the number of prime components in $K$. In the case of prime knot types, $N_{K}=1$, and for the unknot $N_{K}=0$ (see reference $[26,29-31]$ ).

This may be understood as follows: The average structure of a knotted polygon in the scaling limit is that of an unknotted polygon with small knotted ball-pairs distributed along its length. Each knotted ball-pair is an arc of prime knot type with endpoints on a topological ball containing it, and this gives a factor of $n$ for each such ball-pair, giving rise to a factor $n^{N_{K}}$ in equation (36) - see references [30,31] for more details.

Thus, for a polygon of knot type $K$ equation (24) is modified to

$$
\bar{p}_{n}(\vec{r}, K) \simeq C_{K} n^{N_{k}} \sum_{k=0}^{n} P_{k, n-k} C_{k}(\vec{r}) c_{n-k}(\vec{r})
$$

where $\bar{p}_{n}(\vec{r}, K)$ is the number of polygons of length $n$, rooted at $\overrightarrow{0}$, of knot type $K$ with $N_{K}$ prime knot factors, and passing through the vertex $\vec{r}$. The factor 


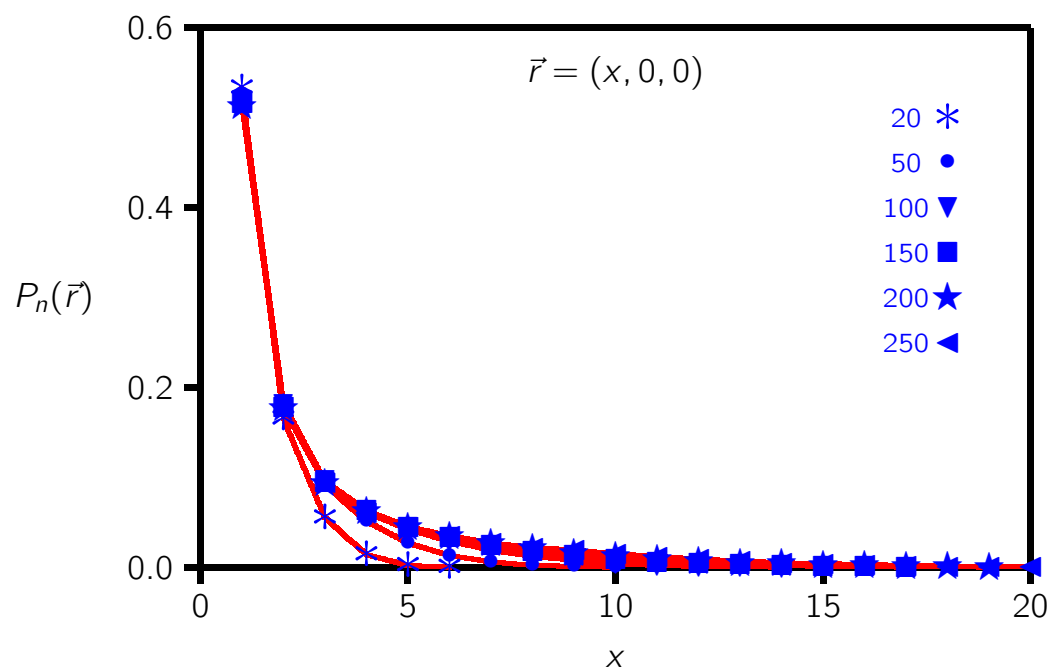

Figure 4: The pressure $P_{n}(x, 0,0)$ (equation (16)) along the $X$-axis at the lattice sites $(x, 0,0)$ for $x \in\{1,2, \ldots, 30\}$, and for $n=20(*), n=50(\bullet), n=100(\boldsymbol{\nabla})$, $n=150(\boldsymbol{\square}), n=200(\star)$ and $n=250(\triangleleft)$.

of $n^{N_{K}}$ is carried through without modification to equation (30). In the case of unknotted polygons $N_{K}=0$, and the pressure has the scaling form given in equation (35). However, if $N_{K} \neq 0$, then the pressure near a knotted polygon is computed from equation (31). Notice that the factor $n^{N_{k}}$ cancels in the numerator and demoninator (which is given by equation (36)). Thus, the scaling of the pressure is unchanged, and for knotted polygons it is given by equation (35).

The GAS algorithm [21] was used to approximately enumerate rooted knotted lattice polygons avoiding fixed vertices $\vec{r}$ in the cubic lattice. The algorithm was tuned to sample polygons of lengths up to $n=400$, sampling along 500 independent sequences, each of length $6 \times 10^{7}$ attempted BFACF moves. This gives a total of $3 \times 10^{10}$ iterations for each knot type in the set $\left\{0_{1}, 3_{1}, 4_{1}, 5_{1}, 5_{2}\right\}$ (where $0_{1}$ is the unknot, $3_{1}$ is the trefoil, and $4_{1}$ is the figure eight knot).

A sample of data for the trefoil knot is shown in table 1 . Data were collected on points in the $x y$-plane of $\mathbb{L}^{3}$ with Cartesian coordinates of the form $(x, y, 0)$ with $0 \leq x \leq 29$ and $1 \leq y \leq 30$. By symmetry this gives estimated pressures in the $x y$-plane for $-30 \leq x \leq 30$ and $-30 \leq y \leq 30$.

\subsection{The pressure of the unknot}

The pressures for the unknot were computed from data for unknotted polygons similar to the data in table 1 by using equation (16). In figure 4 the pressure of unknotted polygons along points on the $x$-axis is plotted as a function of distance from the origin - that is, $P_{n}(x, 0,0)$ is plotted as a function of $x$ for 


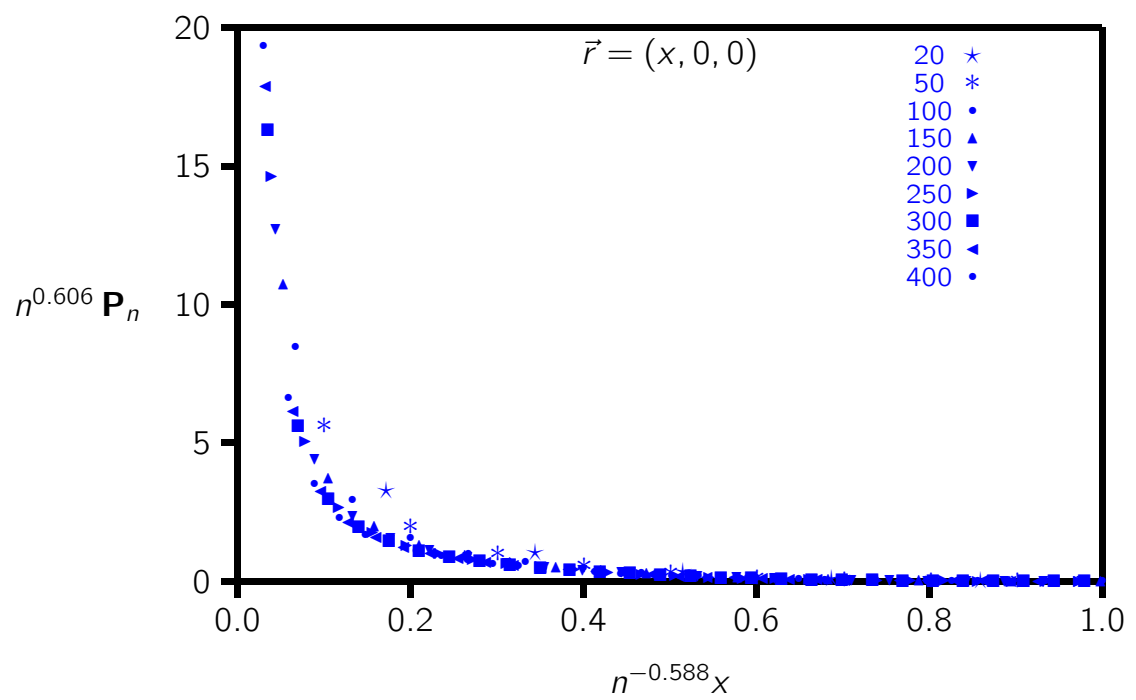

Figure 5: Testing the scaling prediction in equation (35). The rescaled pressure $n^{0.606} P_{n}(x, 0,0)$ is plotted as a function of $n^{-0.588}\|\vec{r}\|_{2}$. These data include all the data points in figure 3. Differently shaped points correspond to different values of $n$, as shown in the key. The data collapse to a single curve, uncovering the scaling function $g_{d}(a) / a^{6}$ in equation (35).

$n \in\{20,50,100,150,200,250\}$.

The data in figure 4 show that the pressure increases quickly on approaching the polygon, which is rooted at the origin $\overrightarrow{0}$ in $\mathbb{L}^{3}$. Since the origin is always occupied by the polygon, the pressure here is infinite (this is an artifact of the model).

Rescaling of the data in figure 4 according to equation (35) shows that $n^{\rho} \mathbf{P}_{n}(a)$ should be plotted against $n^{-\nu}\|\vec{r}\|_{2}$. The scaling analysis of section 2 predicts that this will collapse the data in figure 4 to a single curve.

Using the numerical estimates for $\gamma$ in equation (12) and for $\nu$ in equation (19) gives $\rho \approx 0.606$ (and $\nu \approx 0.588$ ). Thus, the rescaled data $n^{0.606} \mathbf{P}_{n}$ should be plotted against $n^{-0.588}\|\vec{r}\|_{2}$. This is done in figure 5 , which includes all the data in figure 4 as well as additional data from unknotted polygons of length $n \in\{300,350,400\}$. These results show that the data rescale to a single curve with increasing values of $n$ - this is strong evidence in support of the scaling analysis in section 2 .

The plot of the pressure field near an unknotted polygon of length $n=100$ in figure 2 strongly suggests that the field is isotropic. This can be tested numerically by examining the rescaled pressure along other directions in the lattice. In particular, data were collected at points $\vec{r}=(x, x, 0)$ along a diagonal in $\mathbb{L}^{3}$. The resulting plot is illustrated in figure 6 : The data along the two directions collapse to a single curve in the rescaled coordinates, supporting the suggestion that the 


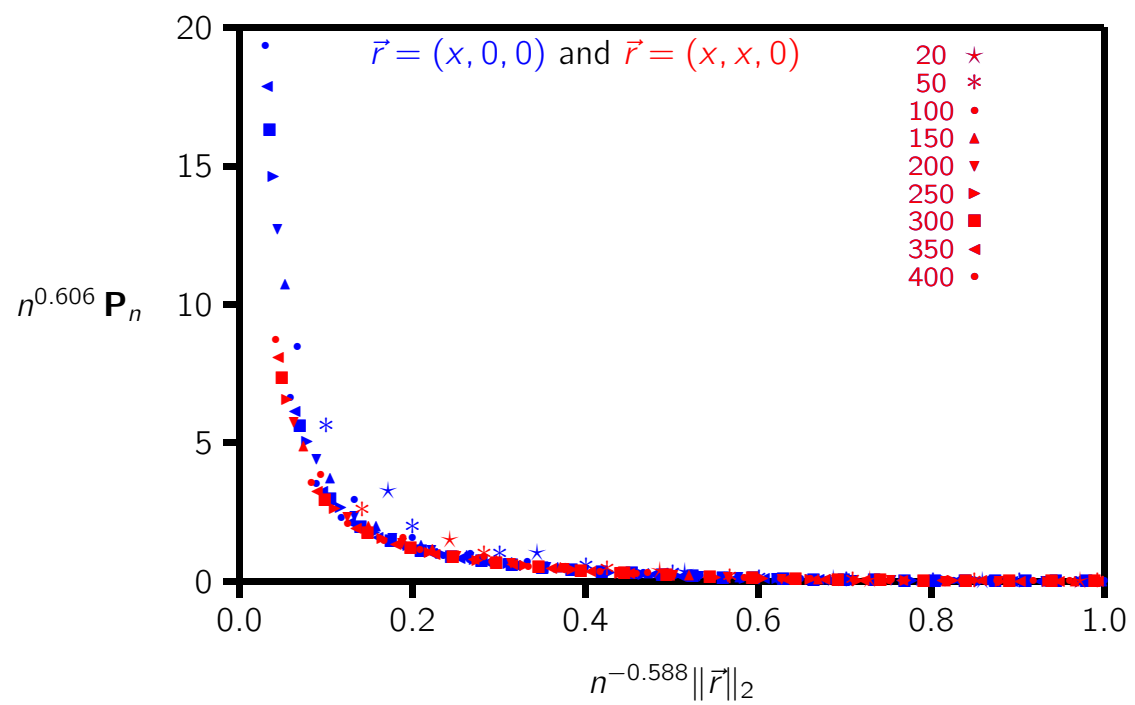

Figure 6: The rescaled pressure along points $\vec{r}=(x, 0,0)$ and $\vec{r}=(x, x, 0)$ for $x \in\{1,2, \ldots, 30\}$. The data of figure 5 are represented as blue points. Red data points represent pressures along the diagonal $\vec{r}=(x, x, 0)$. The data collapse to a single curve, so that the pressure decreases and scales at the same rate along these two directions. This is support for the suggestion in figure 2 that the pressure field is isotropic.

pressure field is isotropic, even at small distances in $\mathbb{L}^{3}$.

The scaling function $g_{d}(a) / a^{6}$ in equation (35) is uncovered in figures 5 and 6. As expected, it decays very quickly with increasing (rescaled) distance. The expected shape of this scaling function is illustrated in figure 3 on logarithmic axes. In figure 7 the data of figure 6 is plotted on log-log axes. The shape of the rescaled data is similar to that of the theoretically derived shape of $g(a) / a^{6}$ shown in figure 3. As expected, the data present a picture of relative high entropic pressure near the lattice polygon, but this crosses through a boundary layer at distance roughly $r \approx n^{\nu}$ (or $a \approx 1$ ) to a phase where the pressure decreases quickly with increasing distance - the crossover happens at the "boundary" of the polygon, which may be considered a gas of monomers localised inside its mean radius.

The turnover at the "boundary" of the polygon defines a "surface layer". At distances inside the surface layer there is a non-zero density of vertices occupied by the lattice knot, giving rise to non-zero entropic pressure which decreases at an exponential rate with increasing distance. Once the surface layer is crossed the rate of decrease in the entropic pressure increases dramatically, and it falls off to zero quickly with increasing distance (as seen in the steep descend of $n^{\rho} \mathbf{P}_{n}$ with increasing distance in figure 7 for $a>1$ ).

A particle approaching the lattice knot from a large distance experiences little pressure at first. The pressure increases steeply as the surface layer of the lattice 


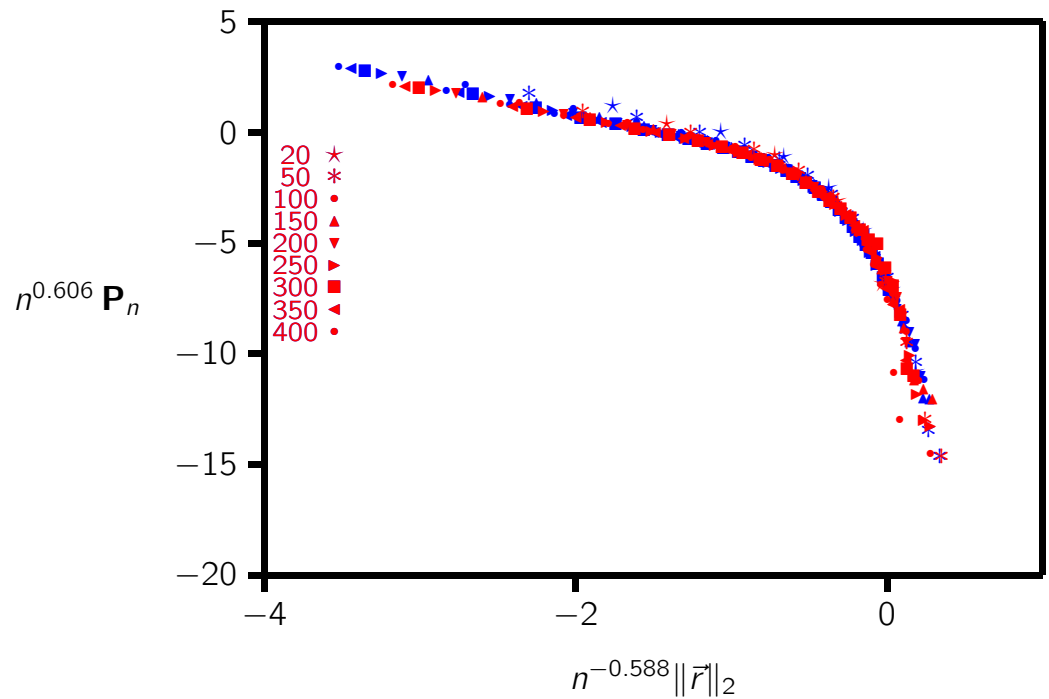

Figure 7: The data of figure 6 on a log-log scale. The rescaled pressure along points $\vec{r}=(x, 0,0)$ are in blue, and along $\vec{r}=(x, x, 0)$ are in red, for $x \in\{1,2, \ldots, 30\}$. The data collapse to a single curve, so that the pressure decreases with distance from the origin in the same way along these directions.

knot is approach. Once the surface layer is crossed the relative pressure gradient reduces to the more gentle slop seen for small values of $a$ in figure 7 - but the increase is still at an exponential rate. The gradient of the pressure induces a repulsive entropic force on an approaching particle - a particle near the lattice knot will be expelled from its vicinity by these induced forces.

In figure 8 all the rescaled data for polygons of lengths $100 \leq 4 n \leq 400$ are plotted on logarithmic axes, for all lattice points $(x, y, 0)$ (where $1 \leq x \leq 30$ and $0 \leq y \leq 29$ ). The data accumulate in the vicinity of a single curve of shape given by figure 3 .

A second graph is presented in figure 9 where the Flory value for $\nu$ (this is $\nu=0.60$ ) was used, and with $\gamma=1.15$. Thus, the rescaled pressure $n^{0.643} \mathbf{P}_{n}(a)$ is plotted against $n^{-0.60}\|x\|_{2}$. The result is a plot very similar to figure 8 , showing that the scaling is also consistent with the Flory value of $\nu$.

\subsection{The pressure near a lattice trefoil}

The entropic pressure near a lattice trefoil knot rooted at the origin were computed at points $(x, 0,0)$ for $1 \leq x \leq 30$ in the same way as data were collected for unknotted polygons. Some data are plotted in figure 10 - note that the shortest polygons have length 24 , which is the minimal length of a cubic lattice trefoil [10].

The data in figure 10 can be rescaled (similar to the unknot data) as was done in figure 5. The result is displayed in figure 11. As for the unknot, the pressure 


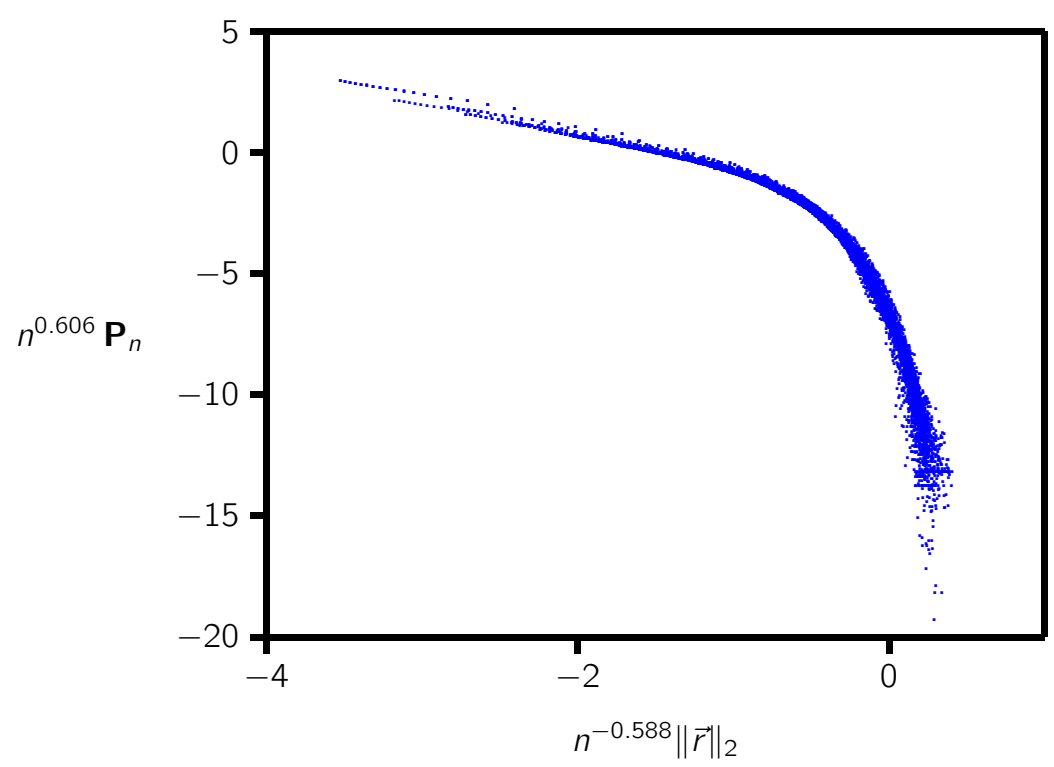

Figure 8: The rescaled pressure $n^{0.606} P_{n}(x, 0,0)$ plotted as a function of $n^{-0.588} x$ on a log-log scale. This scatter plot is for all polygons of lengths $4 n$ with $100 \leq$ $4 n \leq 400$ and for lattice points $(x, y, 0)$ (where $1 \leq x \leq 30$ and $0 \leq y \leq 29$ ). The data uncover the scaling function $\log \left(g_{d}(a) / a^{6}\right)$ (see equation (35)).

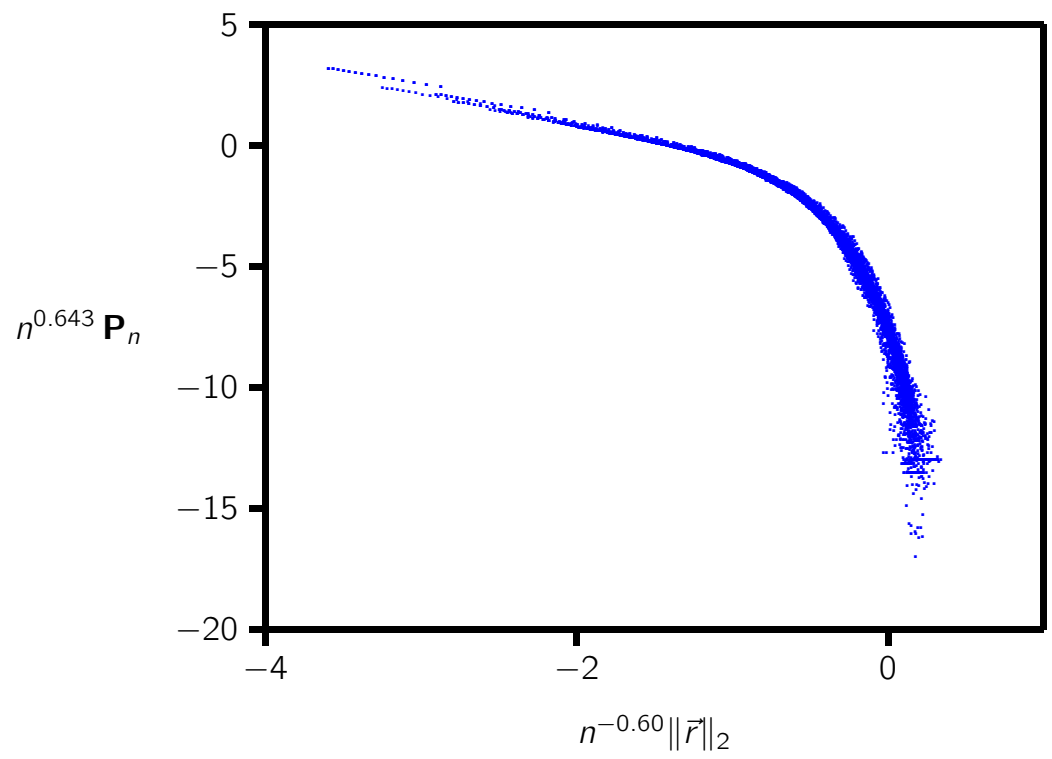

Figure 9: A plot similar to figure 8 , but using the Flory value $\nu=0.60$ instead. 


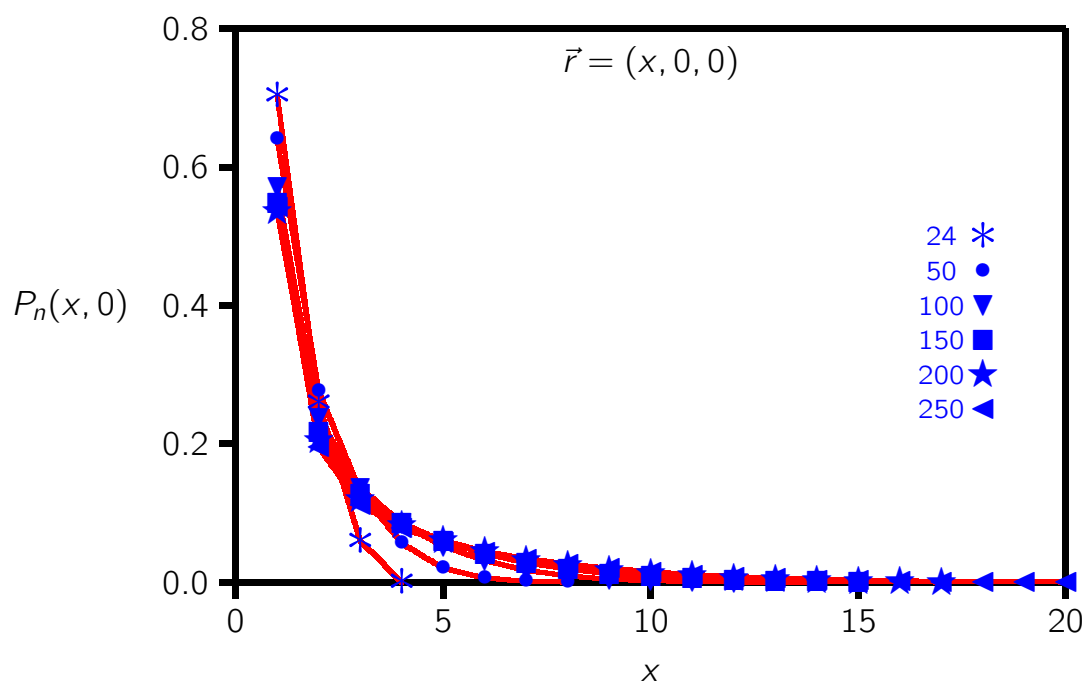

Figure 10: The pressure $P_{n}(x, 0,0)$ of lattice trefoils (equation (16)) along the $X$-axis at the points $(x, 0,0)$ for $x=1,2,3, \ldots, 30$, and for $n=24(*), n=50(\bullet)$, $n=100(\boldsymbol{\Delta}), n=150(\boldsymbol{\square})$ and $n=200(\star)$ and $n=250(\triangleleft)$.

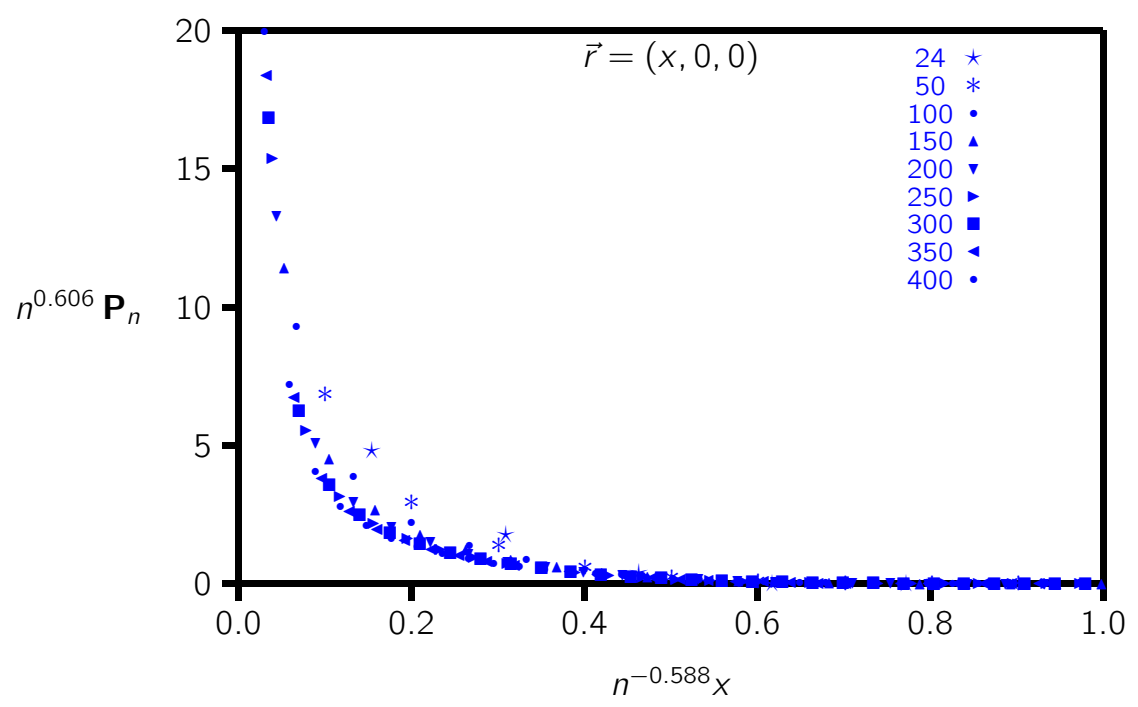

Figure 11: Testing the scaling prediction in equation (35) for lattice trefoils. The rescaling of data in figure 10 is done by plotting $n^{0.606} P_{n}(x, 0,0)$ is plotted as a function of $n^{-0.588} x$. The data collapse to a single curve, uncovering the scaling function $g_{d}(a) / a^{6}$ in equation (35). This plot contains all the data of figure 10 as well as data at larger values of $n$. 


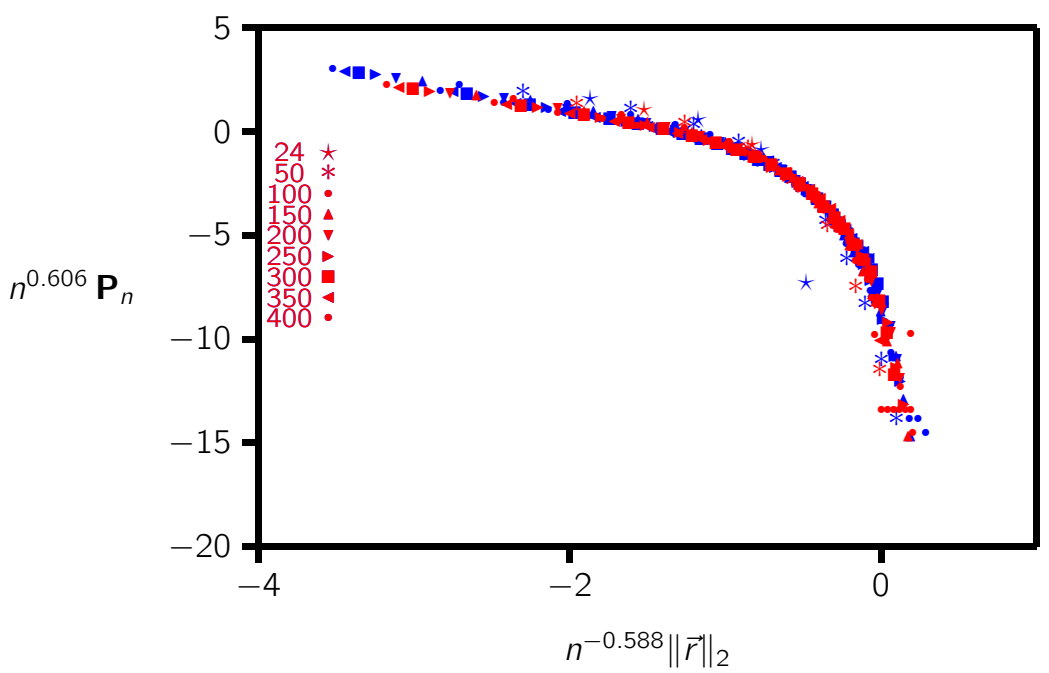

Figure 12: The rescaled pressure $n^{0.606} P_{n}$ plotted as a function of $n^{-0.588}\|\vec{r}\|_{2}$ on a log-log scale for lattice trefoil knots. The data include the pressures at vertices with coordinates $(x, 0,0)$ and $(x, x, 0)$ for $x \in\{1,2, \ldots, 30\}$. This graph also includes all the data in figure 11 . The data collapse to a single curve, uncovering $\log \left(g_{d}(a) / a^{6}\right)$ in equation (35).

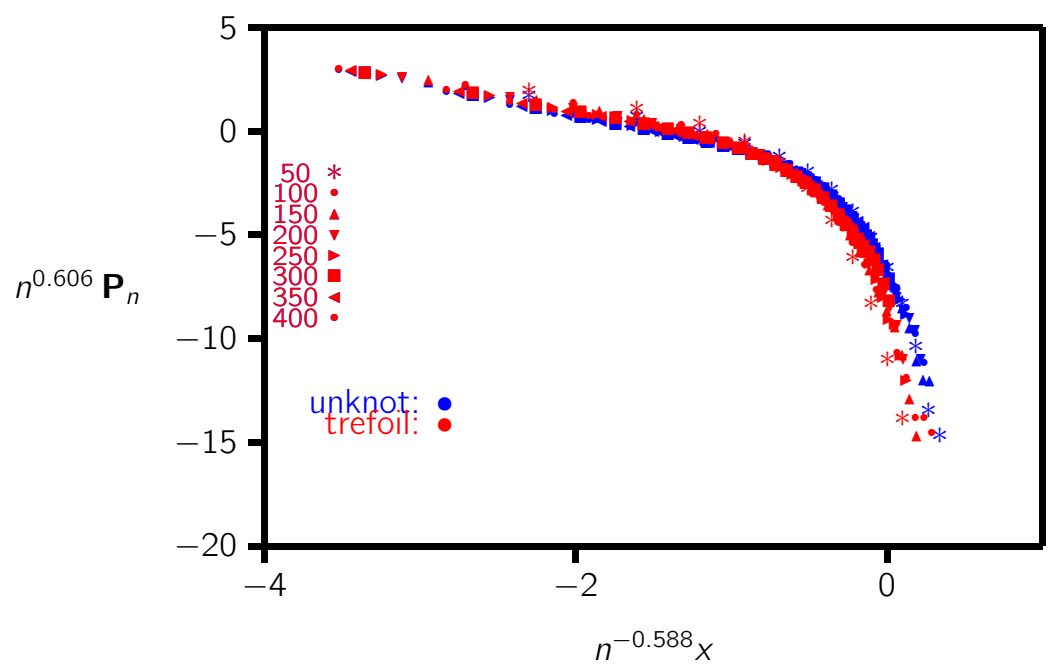

Figure 13: The rescaled pressure $n^{0.606} P_{n}(x, 0,0)$ plotted as a function of $n^{-0.588}\|\vec{r}\|_{2}$ on a log-log scale for the unknot (blue) and the trefoil (red). At small distances the the data coincide, but the points separate for a approaching one, with pressure dropping more quickly for the trefoil than for the unknot. 


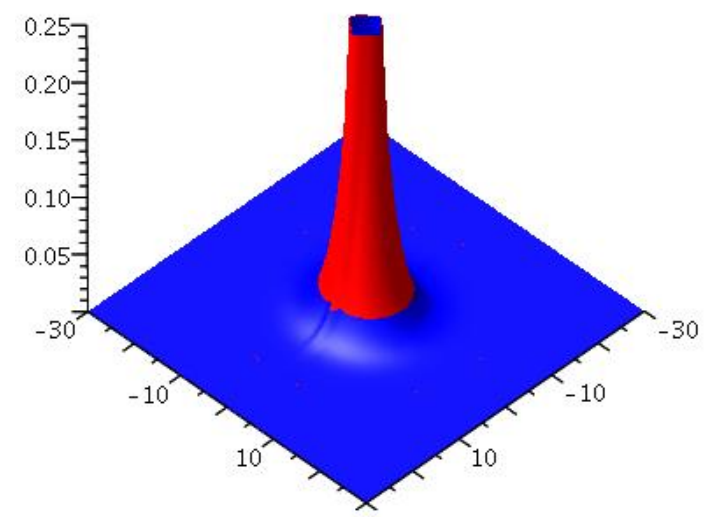

Figure 14: The pressure field near a lattice knots of type unknot and type trefoil plotted on the same graph. Data for the unknot are shown in blue, and for the trefoil knot in red. This shows that the pressure of the trefoil knot is higher close to the origin (at short distances from the knot - this is the red data), and lower than the pressure of the unknot at larger distance (where the blue data is above the red data). This is consistent with the trefoil being a more compact knot, exploring states with vertices closer to the origin (and increasing the pressure there), while not visiting vertices at larger distances at the same rate than the unknotted polygons (therefore lowering the pressure at larger distances).

curves in figure 10 collapse to a single curve, exposing the scaling function for lattice trefoils.

The rescaled pressure of lattice trefoils were also plotted on logarithmic axes in figure 12. This graph includes the pressure on the vertices $(x, 0,0)$ and $(x, x, 0)$ with $1 \leq x \leq 30$. The result is a graph similar to the graph in figure 7 , showing a pressure profile like that of the unknot, with a well-defined surface layer. At the surface layer the relative pressure gradient is large, quickly decaying with distance from the origin - this relative gradient is more gentle once the surface layer is crossed to vertices closer to the origin.

A comparison of the pressures of the unknot and of the trefoil knot may be done by plotting figures 7 and 12 on the same axes. This is done in figure 13, with the blue data points corresponding to the unknot, and the red data points corresponding to the trefoil. The data coincide at short distances, as expected, but as a approaches one the trefoil data falls away marginally more quickly, showing that the trefoil knot is slightly smaller than the unknot, and is therefore less likely to occupy vertices at larger distances from the origin.

In figure 14 the pressures of the unknot and the trefoil are compared for poly- 
gons of length $n=100$. The blue data are identical to the data in figure 2 - this is the pressure of the unknot interpolated at points $(n, m, 0) \in \mathbb{L}^{d}$ for $-30 \leq n, m \leq 30$. The red data are (similarly) the interpolated pressures of polygons of knot type $3_{1}$. At short distances the red data is above the blue, and vice versa at longer distances. This shows that the entropic pressure of a trefoil polygon is higher than that of the unknot at points close to the orgin. At large distances the converse is seen, the unknot has a higher entropic pressure than the trefoil. This result is consistent with the unknot being a more expanded knot, more likely to occupy vertices at relative large distances from the origin, compared to the trefoil.

\subsection{The pressures of other knots}

Simulations were similarly performed to compute the entropic pressure near lattice polygons of knot types $4_{1}, 5_{1}$ and $5_{2}$. These cases should have the same scaling properties as seen for the trefoil (for example in figure 12).

In figure 15 the rescaled entropic pressures of the unknot, the trefoil and the figure eight knot are plotted against the rescaled length $n^{-\nu}\|\vec{r}\|$ for $\vec{r}=(x, 0,0)$ on a log-log scale. The data for the unknot (blue) and the trefoil (red) are the same as in figure 13. Data for the figure eight knot are shown in green. These data show that for small distances the pressures are almost equal. However, at larger distances the data for the figure eight decreases marginally more quickly than for the trefoil. That is, the figure eight knot has smaller pressure as the surface layer is crossed, when compared to the trefoil knot (which in turn has smaller pressure at the surface layer when compared to the unknot).

Similar results are observed when data for $5_{1}$ and $5_{2}$ are added into figure 15 . This is shown in figure 16 - the five crossing knots are slightly more compact than the figure eight knot and so there is a slight excess pressure at small distances and a slight deficit of pressure at larger distances in the surface layer.

The two five crossing knots have (up to the accuracy in this study), virtually the same pressure curve, as seen in figure 17 , but plotting the pressure for polygons of length $n=100$ and knot types $5_{1}$ and $5_{2}$ in figure 18 show that the pressure of $5_{2}$ at short distances exceeds that of $5_{1}$. At longer distances (near the surface layer), $5_{1}$ has higher pressure. The uneven boundary between the green and yellow regimes, and the patches of yellow, are due to noise in the data.

\section{Conclusions}

In this paper the entropic pressure near a self-avoiding walk model of a ring polymer was modelled. This study extends the results in reference [13] to the cubic lattice - the scaling argument in that reference is shown here not to be valid in three dimensions. Instead, a slightly modified approach produced the scaling relation in 


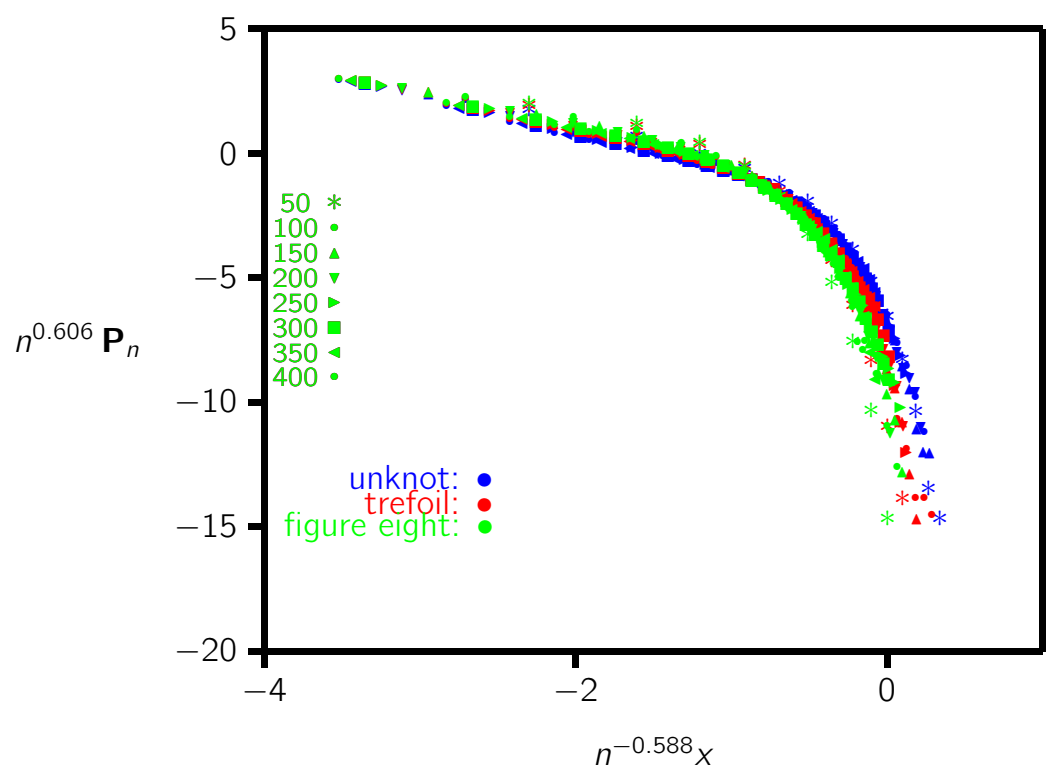

Figure 15: The rescaled pressure $n^{0.606} P_{n}(x, 0,0)$ plotted as a function of $n^{-0.588}\|\vec{r}\|_{2}$ on a log-log scale for the unknot (blue), the trefoil (red) and the figure eight knot (green). At small distances the data scale similarly, with the largest pressure seen for the figure eight knot. At large distances the pressure for the figure eight falls off more quickly than for the trefoil, which in turn decreases more quickly than for the unknot.

equation (33) which may be written as $n^{\rho} \mathbf{P}_{n}(a) \simeq C g_{d}(a) / a^{6}$ where $a$ is length rescaled by $n^{\nu}$ and $\rho=\gamma-d \nu$ in $d \geq 3$ dimensions.

This scaling relation was tested by collecting data on unknotted polygons rooted at the origin in $\mathbb{L}^{3}$. The data gave results consistent with the scaling relation, as seen in figures 5, 6, 7 and 8 . In fact, the data in figures 7 and 8 uncover the predicted shape of the scaling function $g_{d}(a) / a^{6}$ plotted in figure 3 .

Similar results were obtained for knotted polygons. The expectation is that the pressure of a knotted polygon should have the same scaling behaviour as seen for the unknot, and figures 11 and 12 show this to be the case.

A comparison of the rescaled data between the unknot and the trefoil in figure 13 shows minor differences in the rescaled pressure between these knot types. It is not clear that this is due to small corrections to scaling, but the result is consistent with trefoil knots having small relative excess rescaled pressures at short distances from the origin, and small deficit rescaled pressures at longer at the surface layer, compared to the unknot. This pattern is also seen in figures 15 and 16, where more complicated knot types are examined. Those results suggest that more complicated knots have increased pressure close to the origin, and a reduced or a deficit of pressure near and in the surface layer. 


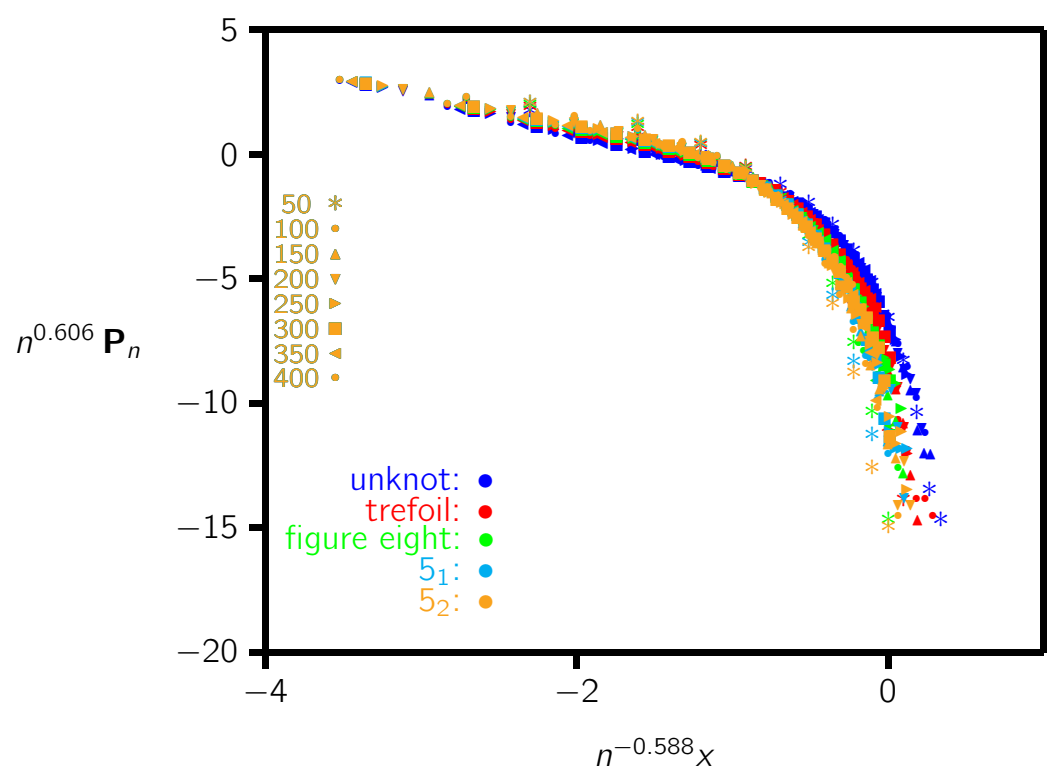

Figure 16: The rescaled pressure $n^{0.606} P_{n}(x, 0,0)$ plotted as a function of $n^{-0.588}\|\vec{r}\|_{2}$ on a log-log scale for the knot types $5_{1}$ (cyan) and $5_{2}$ (orange).

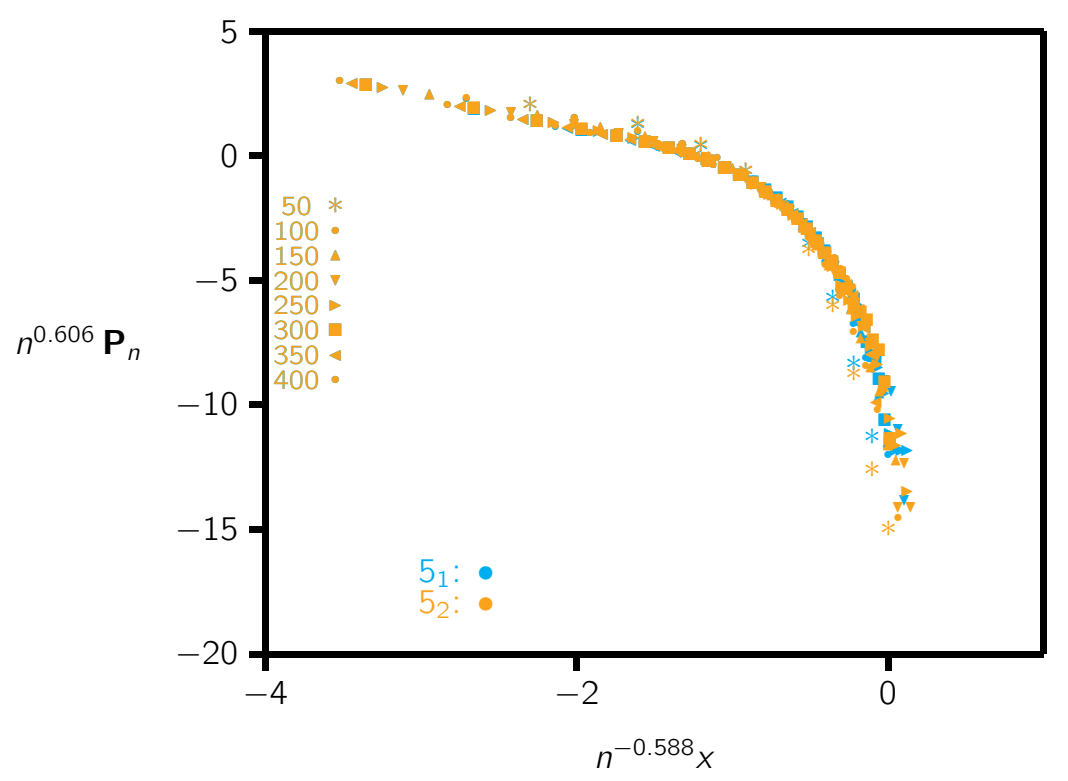

Figure 17: The rescaled pressure $n^{0.606} P_{n}(x, 0,0)$ plotted as a function of $n^{-0.588}\|\vec{r}\|_{2}$ on a log-log scale for the knot types $5_{1}$ (cyan) and $5_{2}$ (orange). 


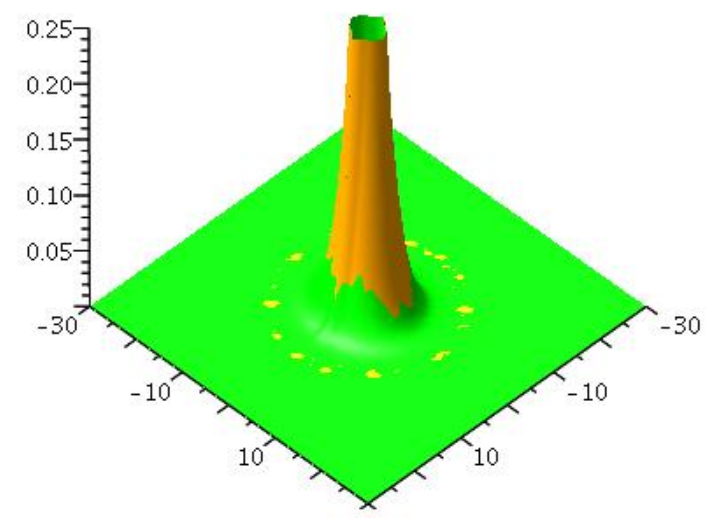

Figure 18: The pressure cone for the knots $5_{1}$ (green) and $5_{2}$ (yellow) near rooted polygons of length $n=100$. These data show that the pressure due to $5_{2}$ exceeds that of $5_{1}$ at short distances, but that $5_{1}$ has larger pressure at longer distances. The uneven barrier between the colours, and the patches of yellow are due to noise in the data.

The results in this study can be extended to models of linear polymers using the same approaches. A more complex situation may be encountered if the polymer is grafted to a hard wall. These are ongoing projects and the results will be forth-coming.

Acknowledgements: EJJvR acknowledges financial support from NSERC (Canada) in the form of a Discovery Grant.

\section{References}

[1] C. Aragao de Carvalho and S. Caracciolo. A new Monte-Carlo approach to the critical properties of self-avoiding random walks. Journal de Physique, 44:323-331, 1983.

[2] B. Berg and D. Foerster. Random paths and random surfaces on a digital computer. Physics Letters B, 106:323-326, 1981.

[3] T. Bickel, C. Marques, and C. Jeppesen. Pressure patches for membranes: The induced pinch of a grafted polymer. Physical Review E, 62(1):11241127, 2000. 
[4] H.D. Bijsterbosch, V.O. De Haan, A.W. De Graaf, M. Mellema, F.A.M. Leermakers, M.A. Cohen Stuart, and A.A. van Well. Tethered adsorbing chains: Neutron reflectivity and surface pressure of spread diblock copolymer monolayers. Langmuir, 11(11):4467-4473, 1995.

[5] R. Brak, A.L. Owczarek, A. Rechnitzer, and S.G. Whittington. A directed walk model of a long chain polymer in a slit with attractive walls. Journal of Physics A: Mathematical and General, 38:4309-4325, 2005.

[6] M.A. Carignano and I. Szleifer. On the structure and pressure of tethered polymer layers in good solvent. Macromolecules, 28(9):3197-3204, 1995.

[7] N. Clisby. Accurate estimate of the critical exponent $\nu$ for self-avoiding walks via a fast implementation of the pivot algorithm. Physical Review Letters, 104:055702, 2010.

[8] J. des Cloizeaux. Lagrangian theory for a self-avoiding random chain. Physical Review A, 10(5):1665-1669, 1974.

[9] J. des Cloizeaux. Short range correlation between elements of a long polymer in a good solvent. Journal de Physique, 41(3):223-238, 1980.

[10] Y. Diao. Minimal knotted polygons on the cubic lattice. Journal of Knot Theory and its Ramifications, 2:413-425, 1993.

[11] B. Duplantier. Statistical mechanics of polymer networks of any topology. Journal of Statistical Physics, 54:581-680, 1989.

[12] M.E. Fisher. Shape of a self-avoiding walk or polymer chain. The Journal of Chemical Physics, 44:616-622, 1966.

[13] F. Gassoumov and E.J. Janse van Rensburg. The entropic pressure of a lattice polygon. Journal of Statistical Mechanics: Theory and Experiment, 2013(10):P10005, 2013.

[14] A. Gholami, J. Wilhelm, and E. Frey. Entropic forces generated by grafted semiflexible polymers. Physical Review E, 74(4):041803, 2006.

[15] R. Guida and J. Zinn-Justin. Critical exponents of the $n$-vector model. Journal of Physics A: Mathematical and General, 31:8103-8122, 1998.

[16] J.M. Hammersley. The number of polygons on a lattice. Proceedings of the Cambridge Philosophical Society, 57:516-523, 1961.

[17] J.M. Hammersley and K.W. Morton. Poor man's Monte Carlo. Journal of the Royal Statistical Society., Series B (Methodological):23-38, 1954.

[18] J.M. Hammersley and D.J.A. Welsh. Further results on the rate of convergence to the connective constant of the hypercubical lattice. The Quarterly Journal of Mathematics, 13(1):108-110, 1962. 
[19] R. Hegger and P. Grassberger. Chain polymers near an adsorbing surface. Journal of Physics A: Mathematical and General, 27:4069-4081, 1994.

[20] E.J. Janse van Rensburg. Approximate enumeration of self-avoiding walks. In Algorithmic Probability and Combinatorics, volume 520 of Contemporary Mathematics. AMS, 2010.

[21] E.J. Janse van Rensburg and A. Rechnitzer. Generalised atmospheric sampling of self-avoiding walks. Journal of Physics A: Mathematical and Theoretical, 42(33):335001, 2009.

[22] E.J. Janse van Rensburg and A. Rechnitzer. Minimal knotted polygons in cubic lattices. Journal of Statistical Mechanics: Theory and Experiment, 2011(09):P09008, 2011.

[23] E.J. Janse van Rensburg and A. Rechnitzer. The compressibility of minimal lattice knots. Journal of Statistical Mechanics: Theory and Experiment, 2012:P05003, 2012.

[24] I. Jensen, W.G. Dantas, C.M. Marques, and J.F. Stilck. Pressure exerted by a grafted polymer on the limiting line of a semi-infinite square lattice. Journal of Physics A: Mathematical and Theoretical, 46(11):115004, 2013.

[25] J.C. Le Guillou and J. Zinn-Justin. Accurate critical exponents from field theory. Journal de Physique, 50:1365-1370, 1989.

[26] B. Marcone, E. Orlandini, A.L. Stella, and F. Zonta. What is the length of a knot in a polymer? Journal of Physics A: Mathematical and General, 38:L15-L21, 2004.

[27] C.M. Mate and V.J. Novotny. Molecular conformation and disjoining pressure of polymeric liquid films. The Journal of Chemical Physics, 94:8420-8437, 1991.

[28] K.M. Middlemiss, G.M. Torrie, and S.G. Whittington. Excluded volume effects in the stabilization of colloids by polymers. The Journal of Chemical Physics, 66:3227-3232, 1977.

[29] E. Orlandini, A.L. Stella, and C. Vanderzande. The size of knots in polymers. Physical biology, 6:025012, 2009.

[30] E. Orlandini, M.C. Tesi, E.J. Janse van Rensburg, and S.G. Whittington. Entropic exponents of lattice polygons with specified knot type. Journal of Physics A: Mathematical and General, 29:L299-L304, 1996.

[31] E. Orlandini, M.C. Tesi, E.J. Janse van Rensburg, and S.G. Whittington. Asymptotics of knotted lattice polygons. Journal of Physics A: Mathematical and General, 31:5953-5968, 1998. 
[32] P. Pincus. Colloid stabilization with grafted polyelectrolytes. Macromolecules, 24(10):2912-2919, 1991.

[33] R. Scharein, K. Ishihara, J. Arsuaga, Y. Diao, K. Shimokawa, and M. Vazquez. Bounds for the minimum step number of knots in the simple cubic lattice. Journal of Physics A: Mathematical and Theoretical, 42:475006, 2009.

[34] R.D. Schram, G.T. Barkema, and R.H. Bisseling. Exact enumeration of selfavoiding walks. Journal of Statistical Mechanics: Theory and Experiment, 2011:P06019, 2011. 